\title{
A Commonsense Patient-Centered Approach to Multimodal Analgesia Within Surgical Enhanced Recovery Protocols
}

This article was published in the following Dove Press journal:

Journal of Pain Research

\author{
Edward R Mariano (D) ${ }^{1,2}$ \\ Michael E Schatman (iD) 3,4 \\ 'Department of Anesthesiology, \\ Perioperative and Pain Medicine, Stanford \\ University School of Medicine, Stanford, \\ CA, USA; ${ }^{2}$ Anesthesiology and \\ Perioperative Care Service, Veterans \\ Affairs Palo Alto Health Care System, \\ Palo Alto, CA, USA; ${ }^{3}$ Department of \\ Public Health \& Community Medicine, \\ Tufts University School of Medicine, \\ Boston, MA, USA; ${ }^{4}$ Department of \\ Diagnostic Sciences, Tufts University \\ School of Dental Medicine, Boston, \\ MA, USA
}

"Enhanced recovery" in the context of a surgical patient is both a plan and a goal at the same time. The goal is faster convalescence after surgery with positive outcomes and no complications. The plan takes the form of an enhanced recovery protocol (ERP) or clinical pathway specific to a surgical procedure, a concept pioneered by Professor Henrik Kehlet from Denmark decades ago. ${ }^{1}$ Over time, this basic concept has evolved and has become arguably too complex. With over twenty elements frequently included in modern ERPs, it is not surprising that consistent implementation and adherence are lacking. ${ }^{2}$ Not all of these elements are critical, and a study of an ERP for bowel resection has demonstrated that three are particularly important: 1) laparoscopic (minimally invasive) approach; 2) early termination of intravenous fluid; and 3) early mobilization (getting out of bed). ${ }^{3}$

This last element is critically dependent on effective pain management. Because the objective of achieving analgesia does not exist in a vacuum and must be balanced with the goal of early mobility, today's standard for pain management in the perioperative period is multimodal analgesia or using more than one analgesic agent with different mechanisms of action. ${ }^{4}$ While a single analgesic agent has the potential to provide complete postoperative pain management alone, it often comes at a significant cost. Consider these two extremes: continuous spinal anesthesia and opioid-only analgesia. Continuous spinal anesthesia, which may involve an infusion of local anesthetic into the cerebrospinal fluid, is arguably the most effective regional analgesic technique available. ${ }^{5}$ In the case of lower extremity arthroplasty, a continuous spinal anesthetic will provide complete postoperative pain relief; however, it will also result in complete immobility and prevent the patient from participating in any physical therapy. For the same surgery, opioid-only analgesia may also provide effective pain management, but it may also render the affected patient unconscious, depress respirations, and precipitate nausea, vomiting and constipation, which can also negatively impact postoperative rehabilitation. Additionally, persistent postoperative opioid use has been identified as a common adverse outcome after surgery. ${ }^{6}$ Given these examples, it is clear why multimodal analgesia has become the cornerstone of all ERPs.

Available data suggest that the increasing number of non-opioid analgesic modalities decreases total opioid usage and opioid-related side effects, ${ }^{7}$ and different patterns of non-opioid analgesic utilization have been identified among
Correspondence: Edward R Mariano Anesthesiology and Perioperative Care Service, VA Palo Alto Health Care System, 380 I, Miranda Avenue (I I2A), Palo Alto, CA 94304, USA

Tel + I $650849-0254$

Fax + I $650852-3423$

Email emariano@stanford.edu 
anesthesiologists based on the type of surgery and patient factors. ${ }^{8}$ The scientific mechanisms of postoperative pain have been described elsewhere. ${ }^{9}$ A practical application of a multimodal analgesic strategy should be patient- and procedure-specific and consider the source, transmission, and processing of the expected surgical pain (Figure 1). We present one commonsense approach using total knee arthroplasty (TKA) as an example below written in a way to facilitate discussions with patients and family members.

\section{Source of Pain}

TKA is one of the most common surgical procedures in the United States and reliably generates severe pain in most patients. ${ }^{10}$ TKA involves removal of the arthritic surfaces of the knee joint and implantation of manufactured prostheses. Localized pain and inflammation are caused by incising and cauterizing skin, subcutaneous layers, and muscle as well as osteotomies ("bone cutting") of the tibia, femur, and patella and nerve injury. Pain at the surgical site may be relieved by non-steroidal antiinflammatory drugs (NSAIDs) or the more selective cyclooxygenase-2 inhibitors (e.g., celecoxib), local anesthetics administered around the knee joint, and cryotherapy (i.e. applying ice to affected areas) with or without compression. ${ }^{11}$ All surgical patients should be eligible for these interventions unless contraindications exist.

\section{Transmission of Pain}

Afferent pain signals are carried from the surgical site to the central neuraxis via peripheral nerves. For certain types of surgery, regional anesthesia techniques (i.e., targeting one area of the body with local anesthetics) can effectively interrupt nerve transmission from the periphery to the spinal cord. These nerve blocks can provide sufficient anesthesia to make general anesthesia unnecessary for some surgeries (e.g., spinal anesthesia for knee

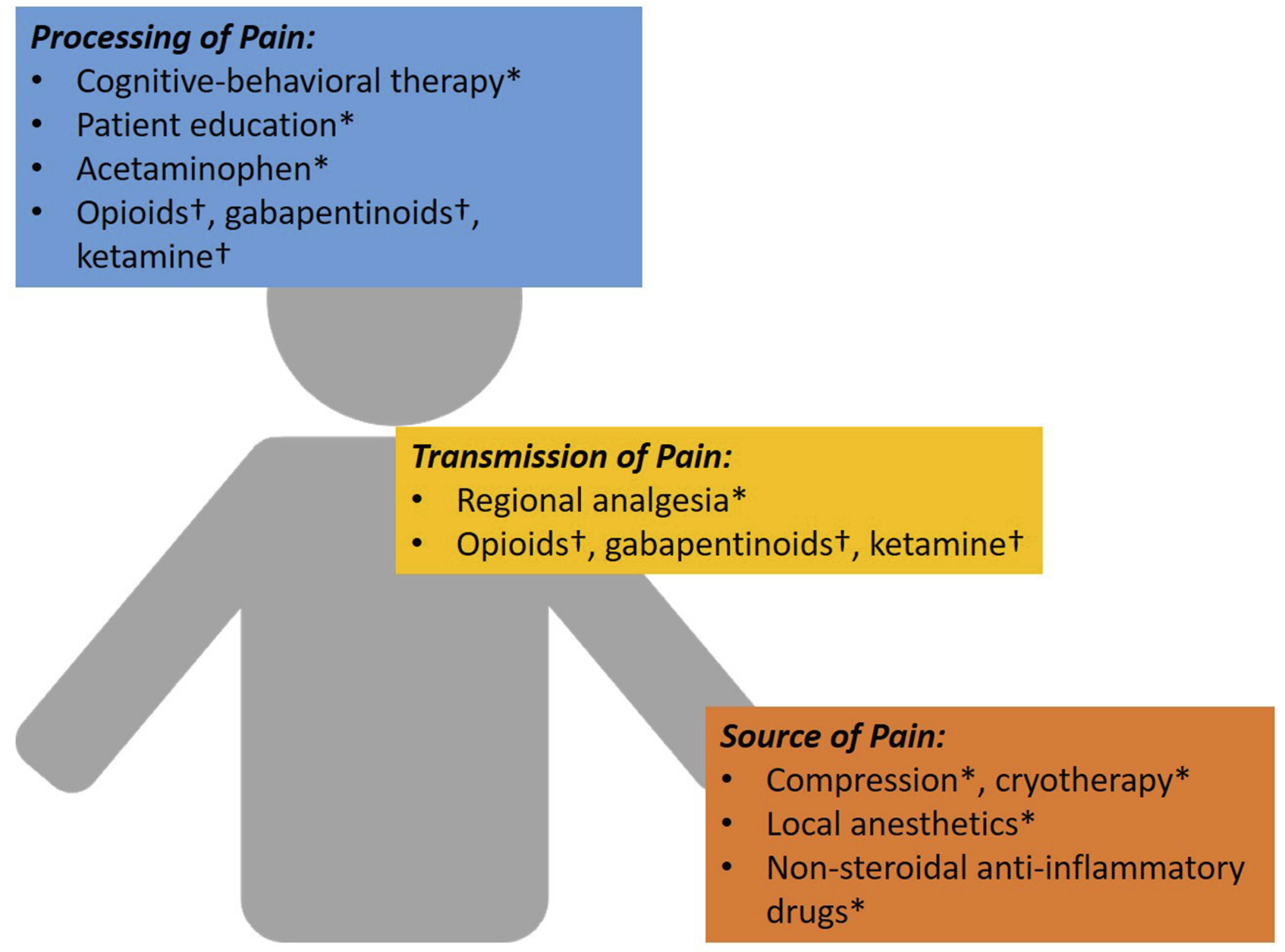

Figure I A simple paradigm for applying a multimodal analgesic strategy taking into consideration the source, transmission, and processing of the expected surgical pain; $*=$ Routine and should be available to all patients unless contraindicated; $\uparrow=$ Not routine and given only as indicated. 
replacement or brachial plexus block for hand surgery) Advances in these techniques using perineural or epidural catheters (i.e., regional analgesia) can extend the duration of target-specific pain relief further into the postoperative period. When this duration matches the expected trajectory of pain resolution after surgery, there may be little need for additional systemic analgesics. For TKA, regional analgesia is well-established as part of the multimodal regimen, ${ }^{4,11}$ but the trajectory of pain resolution may commonly extend beyond the 3-day duration of most regional analgesic techniques. ${ }^{12}$

Another important consideration for patients undergoing major surgery is central sensitization. In simple terms, this entails recruitment of neurons at the level of the spinal cord that normally do not transmit pain and results in expansion of the originally painful area (i.e., hyperalgesia). Opioids indirectly stimulate descending inhibitory pathways in the spinal cord that decrease the transmission of painful signals to the brain. ${ }^{13}$ Gabapentinoids decrease the hyperexcitability of neurons in the spinal cord responsible for central sensitization. ${ }^{14}$ Ketamine acts on N-methyl-D-aspartate receptors and, in subanesthetic doses, has been recommended for acute postoperative pain management. ${ }^{15}$ Further, ketamine may also exert beneficial effects in decreasing central sensitization. ${ }^{16}$ It is our opinion that non-pharmacologic interventions, local anesthetics, and NSAIDs should be administered to all surgical patients who do not have contraindications. However, opioids, gabapentinoids, and ketamine should only be used when indicated. Common factors to consider include patient history, especially comorbid conditions and preoperative analgesic use, preoperative physical function, surgical technique, and the availability of a 24/7 acute pain service that can respond to inadequately treated pain in a timely manner.

\section{Processing of Pain}

Pain processing is both physiological and psychological. By definition, postoperative pain will be an individualized experience. ${ }^{17}$ Previous studies demonstrate that pain catastrophizing is a risk factor for the development of chronic pain following TKA. ${ }^{18}$ For some patients with chronic pain undergoing major surgery, combining cognitivebehavioral therapy with physical therapy may improve postoperative outcomes. ${ }^{19}$ Cognitive-behavioral interventions are time- and labor-intensive and are unfortunately not available everywhere. ${ }^{20}$ Access to and use of basic patient education available online with regard to postoperative analgesic options and safe opioid management are limited. ${ }^{21,22}$ A relatively simple intervention of setting patients' expectations regarding pain after TKA, providing a more detailed pain scale, and educating patients regarding their postoperative pain medications has been shown to decrease the utilization of opioids after surgery. ${ }^{23}$

In terms of pharmacology, paracetamol or acetaminophen acts centrally and should be given to all surgical patients unless contraindicated. Although its exact mechanisms of action are not completely understood, analgesia may result from activation of descending serotonergic (inhibitory) pain pathways, interfering with prostaglandin synthesis, and interacting with the endocannabinoid system. ${ }^{24}$ Other systemic analgesics with supraspinal activity include opioids, gabapentinoids, and ketamine. ${ }^{9,11,13,14,16}$

\section{Putting It All Together}

The implementation of multimodal analgesia within ERPs is best viewed as a checklist and not a recipe. When following a recipe, each ingredient should be included in a recommended amount and added in a stepwise fashion in order to avoid a culinary disaster. This is not the case with multimodal analgesia. Each analgesic item within an ERP should be considered for the eligible surgical patient; included in the regimen if there are no contraindications and/or there are specific indication; and individualized in terms of dosing and frequency based on the patient's prior medication use history and comorbid conditions.

An example of one institution's multimodal analgesic regimen included in an ERP for TKA patients is illustrated in Box 1. While the regimen includes multiple interventions and medications, it may be adapted to meet the needs of the individual patients. ${ }^{4}$ Some patients who are opioidnaïve may not need any scheduled opioid after surgery and will respond to as-need administration only. For all TKA patients, spinal anesthesia is strongly preferred for intraoperative anesthesia ${ }^{25}$ and will eliminate the need for systemic analgesics during surgery. Patients who take opioids chronically before surgery should continue this regimen in the perioperative period. Patients on medication-assisted treatment for opioid use disorder require special consideration with regard to perioperative pain management. ${ }^{26}$ Today, the use of opioids for breakthrough pain relief postTKA has not been eliminated for most patients in the United States even in the setting of robust multimodal analgesia and patient education. ${ }^{23}$ However, the integration of non-pharmacologic interventions and non-opioid 
Box I Sample Perioperative Multimodal Analgesic Regimen Included in an Enhanced Recovery Protocol for Total Knee Arthroplasty Patients

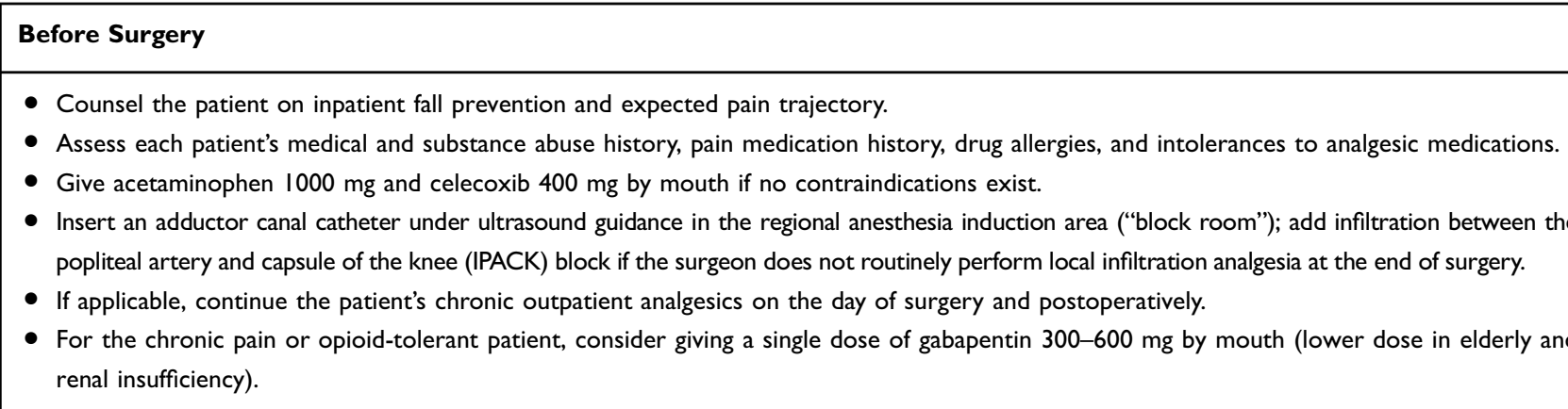

\section{During Surgery}

- Recommend and perform spinal anesthesia (local anesthetic only) unless refused by the patient or contraindicated (then proceed with general anesthesia).

- For the patient who does not receive spinal anesthesia, intravenous opioids may be administered as needed based on reactivity to painful stimuli.

- For the opioid-tolerant patient who receives general anesthesia, consider intraoperative low-dose ketamine infusion $(0.5 \mathrm{mg} / \mathrm{kg}$ bolus followed by $0.25 \mathrm{mg} / \mathrm{kg} / \mathrm{hr}$ infusion).

- Encourage surgeons to perform local infiltration analgesia (ropivacaine $0.2 \% 150 \mathrm{~mL}$ with epinephrine $2.5 \mathrm{mcg} / \mathrm{mL}$ and ketorolac $30 \mathrm{mg}$ ) before closure; if not an option, perform IPACK block preoperatively.

\section{After Surgery}

- Provide routine cryotherapy with or without compression based on institutional practice.

- Prescribe scheduled acetaminophen $1000 \mathrm{mg}$ by mouth every $6 \mathrm{hrs}$ unless contraindicated (reduce the dose in patients with impaired liver function).

- Prescribe scheduled celecoxib $200 \mathrm{mg}$ by mouth twice a day for up to 5 days then continue as needed (avoid if history of gastric ulcer or renal insufficiency).

- Short-acting oxycodone may be prescribed on a scheduled basis 5-10 mg every 6 hrs for the first I-2 days after surgery; replace with the patient's preoperative opioid regimen when applicable.

- For breakthrough pain relief, prescribe short-acting oxycodone tablets (e.g., $5 \mathrm{mg}$ ) by mouth and hydromorphone intravenously (e.g., $0.2 \mathrm{mg}$ ) every 4 hrs as needed.

- Initiate perineural infusion of ropivacaine $0.2 \%$ (basal rate $6 \mathrm{~mL} / \mathrm{hr}$; patient-controlled bolus $5 \mathrm{~mL}$, lockout 30 min) via adductor canal catheter immediately after surgery.

- Provide daily monitoring of the patient's pain experience, achievement of functional goals, and overall recovery through hospital discharge and adjust medications and interventions as needed.

- Counsel the patient on outpatient opioid use and safe storage following discharge and recommend provision of a tapering schedule for prescribed outpatient opioids based on the patient's prior $24 \mathrm{hr}$ opioid use; continue non-pharmacologic interventions and non-opioid analgesics on an as-needed basis.

- For the patient taking opioids chronically before surgery, communicate the discharge analgesic plan with the outpatient prescribing physician.

analgesic modalities including regional anesthesia techniques effectively decreases opioid consumption and should be considered routine. ${ }^{4,11,23}$

In order to be successful, ERPs must be accepted by both healthcare practitioners and patients. Ethical concerns have been raised about the "weaponization" of ERPs with the goal of eliminating all postoperative opioids even in patients on long-term opioids. We appreciate that an important goal of ERPs should be to minimize the need for postoperative opioids whenever possible and practical through effective multimodal analgesia. However, we assert that the intended goal of ERPs, particularly for severely painful surgical procedures (e.g., total joint replacement), is incorporation of "opioid-sparing" rather than "opioid-eliminating" pain management strategies. $^{27}$ Opioids as a class of analgesic drugs continue to have a role in postoperative pain management, and the reality is that many patients presenting for surgery today are already on long-term opioids preoperatively. These patients may rightfully fear "oligoanalgesia" due to the 
deliberate underutilization of opioids in the perioperative period. The ethical application of ERPs emphasizes that pain is a subjective experience, and all ERPs should continue to promote personalized pain medicine in the hospital postoperatively and post-discharge. A recent article describing a multidisciplinary patient-specific opioid prescribing and tapering protocol after lower extremity arthroplasty suggests a reasonable goal of return to baseline opioid use after recovery from surgery. ${ }^{28}$

In conclusion, there has been tremendous progress in the field of acute perioperative pain medicine. Effective pain management to facilitate early mobility is a critical element of modern ERPs for surgical patients. The use of multimodal analgesia has decreased the over-reliance on opioids for postoperative pain relief with regional anesthesia techniques targeting the specific site of pain and systemic non-opioid and non-pharmacologic interventions expanding coverage across the complex network of pain mechanisms. While opioids have yet to be eliminated from the postoperative period, and may well never be, the effectiveness of multimodal analgesia is well proven and has become the standard for perioperative pain management.

\section{Acknowledgments}

This material is the result of work supported with resources and the use of facilities at the Veterans Affairs (VA) Palo Alto Health Care System (Palo Alto, CA, USA). The contents do not represent the views of the Department of Veterans Affairs or the United States Government.

\section{Disclosure}

No financial support was received for this editorial. Dr. Mariano has no conflicts of interest to declare. Dr. Schatman serves as a consultant to Quest Diagnostics, Salix Pharmaceuticals, and Kaleo Pharma. These companies had absolutely no input into the process of preparing this manuscript.

\section{References}

1. Tanious MK, Ljungqvist O, Urman RD. Enhanced recovery after surgery: history, evolution, guidelines, and future directions. Int Anesthesiol Clin. 2017;55(4):1-11. doi:10.1097/AIA.0000000000000167

2. Kehlet H, Joshi GP. Enhanced recovery after surgery: current controversies and concerns. Anesth Analg. 2017;125(6):2154-2155. doi:10.1213/ANE.0000000000002231

3. Pecorelli N, Hershorn O, Baldini G, et al. Impact of adherence to care pathway interventions on recovery following bowel resection within an established enhanced recovery program. Surg Endosc. 2017;31 (4):1760-1771. doi:10.1007/s00464-016-5169-2
4. Schwenk ES, Mariano ER. Designing the ideal perioperative pain management plan starts with multimodal analgesia. Korean $J$ Anesthesiol. 2018;71(5):345-352. doi:10.4097/kja.d.18.00217

5. Ilfeld BM, McCartney CJL. Searching for the optimal pain management technique after knee arthroplasty: analgesia is just the tip of the iceberg. Anesthesiology. 2017;126(5):768-770. doi:10.1097/ ALN.000 0000000001608

6. Brummett CM, Waljee JF, Goesling J, et al. New persistent opioid use after minor and major surgical procedures in US adults. JAMA Surg. 2017;152(6):e170504. doi:10.1001/jamasurg.2017.0504

7. Memtsoudis SG, Poeran J, Zubizarreta N, et al. Association of multimodal pain management strategies with perioperative outcomes and resource utilization: a population-based study. Anesthesiology. 2018;128(5):891-902. doi:10.1097/ALN.0000000000002132

8. Kwong JZ, Mudumbai SC, Hernandez-Boussard T, Popat RA, Mariano ER. Practice patterns in perioperative nonopioid analgesic administration by anesthesiologists in a veterans affairs hospital. Pain Med. 2019. doi:10.1093/pm/pnz226

9. Pogatzki-Zahn EM, Segelcke D, Schug SA. Postoperative pain-from mechanisms to treatment. Pain Reports. 2017;2(2):e588. doi:10.1097/ PR9.0000000000000588

10. Cram P, Lu X, Kates SL, Singh JA, Li Y, Wolf BR. Total knee arthroplasty volume, utilization, and outcomes among medicare beneficiaries, 1991-2010. JAMA. 2012;308(12):1227-1236. doi:10.1001/ 2012.jama.11153

11. Kandarian BS, Elkassabany NM, Tamboli M, Mariano ER. Updates on multimodal analgesia and regional anesthesia for total knee arthroplasty patients. Best Pract Res Clin Anaesthesiol. 2019;33 (1):111-123. doi:10.1016/j.bpa.2019.02.004

12. Lavand'homme PM, Grosu I, France MN, Thienpont E. Pain trajectories identify patients at risk of persistent pain after knee arthroplasty: an observational study. Clin Orthop Relat Res. 2014;472 (5):1409-1415. doi:10.1007/s11999-013-3389-5

13. Pathan H, Williams J. Basic opioid pharmacology: an update. $\mathrm{Br}$ J Pain. 2012;6(1):11-16. doi:10.1177/2049463712438493

14. Chang CY, Challa CK, Shah J, Eloy JD. Gabapentin in acute postoperative pain management. Biomed Res Int. 2014;2014:631756. doi: $10.1155 / 2014 / 631756$

15. Schwenk ES, Viscusi ER, Buvanendran A, et al. Consensus guidelines on the use of intravenous ketamine infusions for acute pain management from the American society of regional anesthesia and pain medicine, the American Academy of Pain Medicine, and the American Society of Anesthesiologists. Reg Anesth Pain Med. 2018;43(5):456-466. doi:10.1097/AAP.0000000000000806

16. De Kock M, Lavand'homme P, Waterloos H. 'Balanced analgesia' in the perioperative period: is there a place for ketamine? Pain. 2001;92 (3):373-380.

17. Pain terms: a list with definitions and notes on usage. Recommended by the IASP subcommittee on taxonomy. Pain. 1979;6(3):249.

18. Burns LC, Ritvo SE, Ferguson MK, Clarke H, Seltzer Z, Katz J. Pain catastrophizing as a risk factor for chronic pain after total knee arthroplasty: a systematic review. J Pain Res. 2015;8:21-32. doi:10.2147/JPR.S64730

19. Archer KR, Devin CJ, Vanston SW, et al. Cognitive-behavioral-based physical therapy for patients with chronic pain undergoing lumbar spine surgery: a randomized controlled trial. J Pain. 2016;17 (1):76-89. doi:10.1016/j.jpain.2015.09.013

20. Knoerl R, Lavoie Smith EM, Weisberg J. Chronic pain and cognitive behavioral therapy: an integrative review. West J Nurs Res. 2016;38 (5):596-628. doi:10.1177/0193945915615869

21. Kumar G, Howard SK, Kou A, Kim TE, Butwick AJ, Mariano ER. Availability and readability of online patient education materials regarding regional anesthesia techniques for perioperative pain management. Pain Med. 2017;18(10):2027-2032. doi:10.1093/pm/ pnw179 
22. Kumar G, Jaremko KM, Kou A, Howard SK, Harrison TK, Mariano ER. Quality of patient education materials on safe opioid management in the acute perioperative period: what do patients find online? Pain Med. 2019. doi:10.1093/pm/pny296

23. Yajnik M, Hill JN, Hunter OO, et al. Patient education and engagement in postoperative pain management decreases opioid use following knee replacement surgery. Patient Educ Couns. 2019;102 (2):383-387. doi:10.1016/j.pec.2018.09.001

24. Anderson BJ. Paracetamol (Acetaminophen): mechanisms of action. Paediatr Anaesth. 2008;18(10):915-921. doi:10.1111/pan.2008.18. issue-10

25. Mudumbai SC, Walters TL, Howard SK, et al. The perioperative surgical home model facilitates change implementation in anesthetic technique within a clinical pathway for total knee arthroplasty. Healthc (Amst). 2016;4(4):334-339. doi:10.1016/j.hjdsi.2016.03.002
26. Harrison TK, Kornfeld H, Aggarwal AK, Lembke A. Perioperative considerations for the patient with opioid use disorder on buprenorphine, methadone, or naltrexone maintenance therapy. Anesthesiol Clin. 2018;36(3):345-359. doi:10.1016/j.anclin.2018.04.002

27. Wainwright TW, Gill M, McDonald DA, et al. Consensus statement for perioperative care in total hip replacement and total knee replacement surgery: enhanced Recovery After Surgery (ERAS $((\mathrm{R})))$ Society recommendations. Acta Orthop. 2019;1-17.

28. Tamboli M, Mariano ER, Gustafson KE, et al. A multidisciplinary patient-specific opioid prescribing and tapering protocol is associated with a decrease in total opioid dose prescribed for six weeks after total hip arthroplasty. Pain Med. 2019. doi:10.1093/pm/ pnz260

\section{Publish your work in this journal}

The Journal of Pain Research is an international, peer reviewed, open access, online journal that welcomes laboratory and clinical findings in the fields of pain research and the prevention and management of pain. Original research, reviews, symposium reports, hypothesis formation and commentaries are all considered for publication. The manuscript management system is completely online and includes a very quick and fair peer-review system, which is all easy to use. Visit http:// www.dovepress.com/testimonials.php to read real quotes from published authors. 